\title{
PEDOMETRIA APLICADA A ESTUDO COMPARATIVO ENTRE MORFINA E TRAMADOL EM BOVINOS
}

\section{PEDOMETRY APPLIED TO A COMPARATIVE STUDY USING MORPHINE AND TRAMADOL IN BOVINE}

\author{
Geison Morel Nogueira1 ORCID - http://orcid.org/0000-0002-0503-6294 \\ Sílvia Helena Venturoli Perri² ORCID - http://orcid.org/0000-0001-5083-5557 \\ Paulo Sérgio Patto Dos Santos² ORCID - http://orcid.org/0000-0002-8535-5569 \\ Celso Antonio Rodrigues ${ }^{3^{*}}$ ORCID - http://orcid.org/0000-0002-4837-463X \\ 1Faculdade de Medicina Veterinária, UFU, Uberlândia, MG, Brasil. \\ 2Faculdade de Medicina Veterinária de Araçatuba, UNESP, Araçatuba, SP, Brasil. \\ 3Faculdade de Medicina Veterinária e Zootecnia, UNESP, Botucatu, SP, Brasil. \\ *Autor para correspondência - rodriguesca@fmvz.unesp.br
}

\section{Resumo}

A analgesia com opioides é limitada ou indefinida em ruminantes. Neste trabalho, objetivou-se a realização de um estudo comparativo entre dois analgésicos opioides: a morfina e o tramadol, com base nas avaliações clínica e pedométrica de animais submetidos a artrite e sinovite experimental transitórias, desenvolvidas na articulação interfalângica distal, após administração intra-articular de anfotericina B. Utilizou-se seis animais, em dois tratamentos distintos, com morfina, na dose de $0,5 \mathrm{mg} / \mathrm{kg}$ e 20 dias depois com tramadol, na dose de $1,8 \mathrm{mg} / \mathrm{kg}$, ambos via intramuscular. Os animais foram avaliados em intervalos de três horas, num total de 27 horas, observando-se parâmetros fisiológicos, deambulação e atividade pedométrica. Claudicação e alterações pedométricas foram observadas para ambos os fármacos. Por meio das variáveis da atividade pedométrica, observou-se um padrão de inquietação compatível com nocicepção podal, não havendo a interferência dos fármacos sobre a claudicação. Concluiu-se que a morfina e o tramadol, nas doses testadas, foram incapazes de interferir na atenuação do grau de claudicação no momento de máxima estimulação dolorosa, frente ao modelo experimental de dor ortopédica.

Palavras-chave: Anfotericina B; artrite; claudicação; dor; sinovite.

\begin{abstract}
The use of opioids for analgesia is limited or undefined in ruminants. The aim of this study was to compare two analgesics opioids, morphine and tramadol, based on the clinical and pedometric evaluations of animals submitted to a temporary experimental arthritis and synovitis, developed at the distal interphalangeal joint after an intra-articular administration of amphotericin B. Six animals were used in two different treatments, using morphine, dose of $0,5 \mathrm{mg} / \mathrm{kg}$, and twenty days later, tramadol, dose of $1,8 \mathrm{mg} / \mathrm{kg}$, both intramuscularly. The animals were evaluated at 3-hour intervals for a total of 27 hours, observing the physiological parameters, ambulation and pedometer activity. Lameness and pedometer variations were observed in both treatments. Based on the variables of pedometric activity, a pattern of restlessness compatible with podal nociception was observed, and there was no interference of any of the drugs on lameness. It was concluded that morphine and tramadol, at the tested doses, were incapable of interfering in the lameness attenuation during the maximum painful stimulation moment in this experimental protocol of orthopedic pain.
\end{abstract}


Keywords: Amphotericin B; arthritis; lameness; pain; synovitis.

Recebido em: 14 de dezembro de 2016.

Aceito em: 1 de abril de 2019.

\section{Introdução}

Apesar de grande parte da literatura atual, na área de clínica de ruminantes, ser dedicada a claudicação com sede na extremidade distal dos membros, muito ainda continua por se esclarecer. Aspectos referentes à apresentação comportamental do bovino, devido à dor associada à claudicação ${ }^{(1)}$ e as práticas de analgesia disponíveis, frente aos processos mórbidos e seus tratamentos na espécie, ainda são assuntos pouco explorados ${ }^{(2)}$.

Metodologias experimentais para o desenvolvimento de dor podal têm sido utilizadas para avaliação da eficácia de fármacos anti-inflamatórios não esteroidais, na melhora da claudicação ${ }^{(1,3)}$. Contudo, tais medicamentos em uso prolongado estão associados a efeitos indesejáveis como úlceras abomasais e nefrotoxicidade ${ }^{(4)}$.

A utilização de analgésicos opioides, como alternativa para o manejo de dor em bovinos, ocorre, principalmente, através da via de administração epidural ${ }^{(5)}$. Contudo, a aplicação sistêmica destes fármacos é limitada a poucos princípios ativos como a morfina, meperidina, buprenorfina e butorfanol ${ }^{(6)}$.

A partir da realização do exame clínico específico e utilização da pedometria, como método de identificação precoce para afecções podais em bovinos, é possível a observação e avaliação da manifestação de dor podal na espécie ${ }^{(7,8)}$. Contudo, o seu controle por meio de alguns fármacos opioides é uma alternativa pouco explorada ${ }^{(4,6)}$.

Este estudo apresenta a hipótese da análise comparativa entre um fármaco opioide utilizado na rotina clínica (morfina) $)^{(4,6)}$ e o proposto (tramadol), utilizando-se a avaliação clínica e pedométrica para observação de eficácia do tramadol como opção terapêutica no controle da dor podal em bovinos.

Objetivou-se a realização de um estudo comparativo entre dois analgésicos opioides: a morfina, a partir de dose analgésica específica para bovinos, e o tramadol, ainda sem utilização terapêutica na espécie; com base nas avaliações clínica e pedométrica, a partir de modelo experimental de dor podal transitória.

\section{Material e Métodos}

A metodologia adotada está em conformidade com os princípios éticos na experimentação animal, aprovada pela Comissão de Ética no Uso de Animais da Faculdade de Medicina Veterinária e Zootecnia - FMVZ, UNESP / Botucatu, sob o protocolo ${ }^{\circ}$ 15/2013.

Para realização do estudo comparativo entre a morfina e o tramadol foram utilizados seis bovinos hígidos, mestiços, machos não castrados, com idades variando entre 24 e 30 meses e pesando em média $230 \pm 13.03 \mathrm{~kg}$. 
Os animais foram mantidos em baias de 4,0x3,5 m², sendo retirados para avaliação de deambulação e exame físico nos tempos estabelecidos e conduzidos ao tronco de contenção para tal. Receberam água, sal mineral ad libitum e silagem de sorgo duas vezes ao dia.

Previamente ao período de adaptação de sete dias, todos os bovinos foram submetidos à avaliação radiográfica das articulações interfalângicas dos membros pélvicos, nas projeções látero-medial e dorso-plantar, utilizando-se aparelho de raios X (Orange, modelo 1060HF), padronizando-se a técnica em 55kVp, 4mAs, DFF de $100 \mathrm{~cm}$, com filme Kodak ${ }^{\circledR}$, tamanho 30x40 cm. Também foram realizados hemograma e coproparasitológico (OPG). Todos estes procedimentos e exames objetivaram comprovar a higidez e ausência de enfermidades ortopédicas na extremidade dos membros pélvicos. Não foi necessária a exclusão de nenhum animal. No momento estipulado para indução a artrite e sinovite transitória (T0), os bovinos foram contidos fisicamente em brete e submetidos a tricotomia e antissepsia da região, imediatamente proximal à borda coronária do dígito lateral do membro a ser avaliado.

A artrite e sinovite foram induzidas em dois momentos distintos, em todos os bovinos, conforme metodologia desenvolvida por Kotschwar et al. ${ }^{(3)}$. Consistiu-se na administração intra-articular de uma dose única de $20 \mathrm{mg}$ de anfotericina $\mathrm{B}$ (Anfotericin $\mathrm{B}{ }^{\circledR}$, Laboratório Cristália, Brasil), mediante artrocentese interfalângica distal do dígito lateral do membro pélvico, utilizando-se agulha hipodérmica descartável 40x12 $\mathrm{mm}$. O posicionamento da agulha para punção foi de $1 \mathrm{~cm}$ proximal a borda coronária do casco e $1 \mathrm{~cm}$ abaxial ao tendão do músculo extensor digital longo, angulada em aproximadamente 60 graus em direção à região da sola (Figura 1).

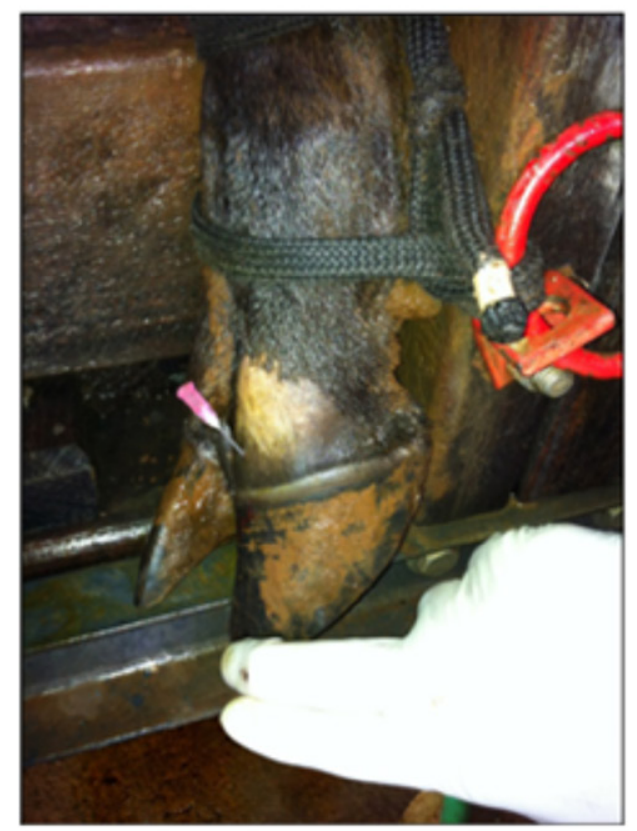

Figura 1. Realização da artrocentese interfalângica distal, após tricotomia e antissepssia, com agulha hipodérmica, momentos antes da administração da anfotericina B.

Os animais foram distribuídos em dois grupos, considerando-se os tratamentos aos quais foram submetidos: morfina (GM) e tramadol (GT). Inicialmente, foram manejados seis animais tratados com sulfato de morfina, após indução de artrite e sinovite interfalângica distal transitória, no dígito lateral do membro pélvico direito. Decorridos 20 dias, deu-se início à segunda etapa, utilizando-se a mesma metodologia no membro pélvico esquerdo, tratando-se os animais com cloridrato de tramadol. Desta 
forma, foram considerados o tempo de evolução clínica e resolução da sinovite e artrite transitórias, induzidas por anfotericina $\mathrm{B}$, de 72 horas $^{(1)}$.

A avaliação clínica foi procedida por meio da aferição das frequências cardíaca, respiratória, motilidade ruminal, e determinação visual do escore de claudicação.

Nos momentos de avaliação estipulados para o estudo, os animais foram conduzidos ao passo, das baias ao tronco de contenção, onde, decorridos três a cinco minutos de aclimatação em brete, foram aferidas as frequências cardíaca e respiratória. A motilidade ruminal foi auscultada em período de cinco minutos.

Para avaliação de claudicação, utilizou-se escore de 0 a 4, segundo Desrochers et al.(7), sendo: grau 0 - indicativo de ausência de claudicação; grau 1 - claudicação leve, com arqueamento de dorso e discreta alteração na locomoção; grau 2 - claudicação moderada, com arqueamento de dorso e locomoção alterada; grau 3 - claudicação intensa, com arqueamento de dorso e claudicação marcante; grau 4 -imobilidade, com relutância em se locomover e grande dificuldade de apoio.

Os animais foram observados em deslocamento ao passo, de forma individual, da baia até o tronco de contenção, pelo menos 20 metros em linha reta e seus retornos. O local era constituído em piso de concreto, sem inclinação, obstáculos ou detritos.

A pedometria foi realizada com a aplicação de pedômetro (Pedometer Plus Tags ${ }^{\circledR}$, AfiMilk TM, Israel) na região metatarsiana do membro pélvico contralateral ao submetido à indução da artrite e sinovite. Com este equipamento, foram colhidos dados por leitura digital, referentes ao número total de passos, quantidade de vezes em que o animal se posicionou em decúbito e tempo em que permaneceu nessa posição, expresso em minutos $^{(8)}$ e números de toques no solo (passos falsos).

Todos os bovinos receberam os pedômetros e foram submetidos a um período de adaptação de sete dias, sendo mantidos em baias individualmente e manejados mimetizando-se as atividades desenvolvidas durante o experimento.

Os valores de referência, utilizados para pedometria inicial (T0), foram coletados considerando-se o intervalo de tempo que antecedeu o início do experimento em três horas (T-3), no qual os valores foram realizados a partir da subtração da leitura final pela inicial. Realizou-se a aferição dos valores de atividade pedométrica, assim como a mensuração de parâmetros fisiológicos neste momento (T0), com a finalidade de se estabelecer um padrão para os dados de: número de decúbitos, tempo de decúbito, números de passos e número de toques ao solo, frequências cardíaca e respiratória, e motilidade ruminal, sem a estimulação dolorosa podal.

As mensurações pedométricas e avaliação clínica, ocorreram em intervalos de três horas, durante um período total de 27 horas.

Os momentos de avaliação e tratamento, foram determinados com base no modelo experimental de dor podal transitória, induzida por anfotericina B intra-articular ${ }^{(1,3)}$.

A metodologia utilizada segue a apresentação esquemática da linha do tempo a seguir:

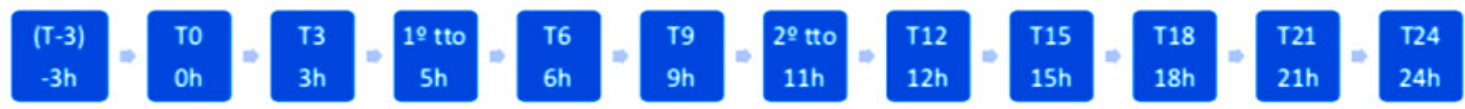

Onde: (T-3) foi o momento basal, para início da contagem de atividade pedométrica, permitindo 
assim que em T0 houvesse a leitura de valores de pedometria, assim como mensuração de parâmetros fisiológicos, sem estimulação dolorosa podal. Em T0 também ocorrem a indução a artrite e sinovite transitórias. O tto foi referente aos momentos de aplicação da morfina ou tramadol. As mensurações das variáveis ocorreram em intervalos de três horas ao longo de 27 horas.

O GM foi tratado com o sulfato de morfina (Dimorf®, Laboratório Cristália, Brasil). O fármaco foi administrado pela via intramuscular, totalizando duas aplicações, com intervalo de seis horas, na dose de $0,5 \mathrm{mg} / \mathrm{kg}^{(4,6)}$. A primeira aplicação de morfina foi realizada cinco horas após a indução da artrite e sinovite, uma hora antes da evidenciação dolorosa podal, que ocorre no momento de aproximadamente seis horas após administração intra-articular de anfotericina $\mathrm{B}$, de acordo com modelo experimental ${ }^{(3)}$.

Foram aferidos parâmetros vitais, procedida a leitura do pedômetro e avaliação visual de claudicação no momento da indução no tempo zero, bem como nos momentos subsequentes em 3, 6, 9, 12, 15, 18,21 e 24 horas.

O grupo tramadol (GT), composto pelos mesmos seis animais, recebeu cloridrato de tramadol (Tramadon ${ }^{\circledR}$, Laboratório Cristália, Brasil), após a realização do mesmo protocolo de indução a artrite e sinovite. Contudo, fazendo uso do membro pélvico esquerdo e decorridos 20 dias do protocolo inicial, após completa recuperação dos animais. O fármaco foi administrado uma única vez, pela via intramuscular, na dose de $1,8 \mathrm{mg} / \mathrm{kg}$.

A dose e frequência de aplicação experimental do tramadol, foram determinados por cálculo de extrapolação alométrica ${ }^{(9)}$. O tratamento também foi iniciado cinco horas após a indução do processo inflamatório articular, do dígito lateral no membro pélvico. Decorridas seis horas da administração do tramadol, foi realizada administração de solução fisiológica, em igual volume, via intramuscular, mimetizando a segunda dose do analgésico, administrada ao GM.

A análise estatística foi realizada empregando-se o programa Statistical Analysis System, versão 9.3, com o intuito de se verificar a existência de alterações entre as variáveis obtidas entre os grupos GT e GM, além do comportamento das variáveis ao longo dos momentos avaliados dentro de cada grupo.

As variáveis: frequência cardíaca; frequência respiratória; número de toques no solo e tempo de decúbito apresentaram distribuição normal de acordo com o teste de Shapiro-Wilk e foram submetidas à análise de variância com medidas repetidas (ANOVA) e as médias comparadas pelo teste de Tukey.

O número de decúbitos, número de passos e motilidade ruminal não apresentaram valores de distribuição normal. Assim as variáveis: número de passos; número de decúbitos; motilidade ruminal e o grau de claudicação foram avaliados pelo teste de Wilcoxon para comparar os grupos em cada momento e teste de Friedman para comparar os momentos dentro de cada grupo e as medianas comparadas pelo teste de Dunn. Os resultados foram considerados significativos quando $\mathrm{P}<0,05$. As análises estatísticas foram efetuadas empregando-se o programa Statistical Analysis System, versão 9.3 .

\section{Resultados}

A aferição das frequências cardíaca e respiratória dos grupos, indicou valores que se mantiveram em torno dos limites superiores dos fisiológicos para a espécie ${ }^{(10)}$ (Tabela 1), não havendo diferença significativa entre os tempos de avaliação. 
Tabela 1. Média $(\bar{x})$ e desvio padrão (S) das frequências cardiaca, em bpm, e respiratória, em mpm, segundo o grupo e tempo de avaliação

\begin{tabular}{ccccc}
\hline \multirow{2}{*}{ Tempo (h) } & \multicolumn{2}{c}{ Frequência cardíaca } & \multicolumn{2}{c}{ Frequência respiratória } \\
\cline { 2 - 5 } & GM & GT & GM & GT \\
\cline { 2 - 5 } & $\bar{x} \pm \mathbf{S}$ & $\bar{x} \pm \mathbf{S}$ & $\bar{x} \pm \mathbf{S}$ & $\bar{x} \pm \mathbf{S}$ \\
\hline 0 & $70 \pm 8,6$ & $75 \pm 6,5$ & $37 \pm 7,1^{*}$ & $24 \pm 4,4^{\mathrm{ab}}$ \\
3 & $79 \pm 8,4$ & $74 \pm 14,9$ & $26 \pm 11,2$ & $20 \pm 3,6^{\mathrm{b}}$ \\
6 & $85 \pm 10,6^{*}$ & $71 \pm 9,3$ & $28 \pm 8,0$ & $26 \pm 6,6^{\mathrm{ab}}$ \\
9 & $83 \pm 13,8^{\circ}$ & $78 \pm 20,4$ & $30 \pm 6,1$ & $29 \pm 7,3^{\mathrm{ab}}$ \\
12 & $82 \pm 5,8$ & $83 \pm 18,0$ & $31 \pm 7,4$ & $28 \pm 8,0^{\mathrm{ab}}$ \\
15 & $81 \pm 14,0$ & $76 \pm 13,0$ & $34 \pm 6,0$ & $30 \pm 3,6^{\mathrm{a}}$ \\
18 & $85 \pm 10,0$ & $75 \pm 7,5$ & $26 \pm 6,1$ & $27 \pm 4,3^{\mathrm{ab}}$ \\
21 & $87 \pm 11,2^{*}$ & $70 \pm 7,8$ & $26 \pm 4,2$ & $28 \pm 2,8^{\mathrm{ab}}$ \\
24 & $77 \pm 10,7$ & $72 \pm 6,1$ & $25 \pm 6,4$ & $26 \pm 4,7^{\mathrm{ab}}$ \\
\hline
\end{tabular}

${ }^{\text {sb }}$ Médias seguidas de letras distintas na coluna diferem entre si pelo teste de Tukey $(\mathrm{P}<0,05)$

*Existe diferença significativa entre os grupos $(\mathrm{P}<0,05)$

Para motilidade ruminal, registrou-se a redução dos valores fisiológicos em todos os momentos, inclusive no inicial (T0), em que foram auscultados dois movimentos em dois minutos, e um movimento em dois minutos para os demais tempos de avaliação.

Apesar de não serem encontradas diferenças significativas entre os grupos GM e GT na variável escore de claudicação (Figura 2), observou-se a ocorrência de claudicação grau 3, significativamente maior quando comparada entre os tempos seis a 12 horas, após a indução do estímulo doloroso podal.

Ressalta-se que esta evidenciação de dor, através da manifestação da claudicação grau 3, iniciou uma hora após o uso dos analgésicos, mantendo-se em até sete horas após. Tal intervalo, coincide com o momento de máxima manifestação dolorosa, segundo modelo experimental de dor podal transitória, entre seis e 12 horas após a indução de artrite e sinovite interfalângica(1).

Houve redução da claudicação em 15 a 24 horas (Figura 2), sendo observada a ausência deste sinal clínico somente no tempo zero em ambos os grupos e em até três horas após no GT.

Clinicamente, a claudicação esteve presente até o término do experimento, contudo, nenhum animal atingiu claudicação grau 4 .

De forma complementar a aferição do grau de claudicação, foram mensurados os valores de atividade pedométrica: número de decúbitos, tempo de decúbito, número de passos e número de toques no solo.

Em relação ao número de decúbitos, na Tabela 2, observou-se diferença significativa entre os grupos em 12 horas, sendo o maior valor da variável para o GT.

No GM o maior número de vezes em que os animais estiveram em decúbito ocorreu em seis horas, uma hora após a primeira administração do fármaco. Observou-se ainda que no momento 12 horas, uma hora após a segunda aplicação, não houve diferença significativa desta variável. O valor mínimo, significando a ausência deste posicionamento, ocorreu em três, 15 e 18 horas. 
No GT observou-se o maior valor em nove horas e em 15 horas o menor.

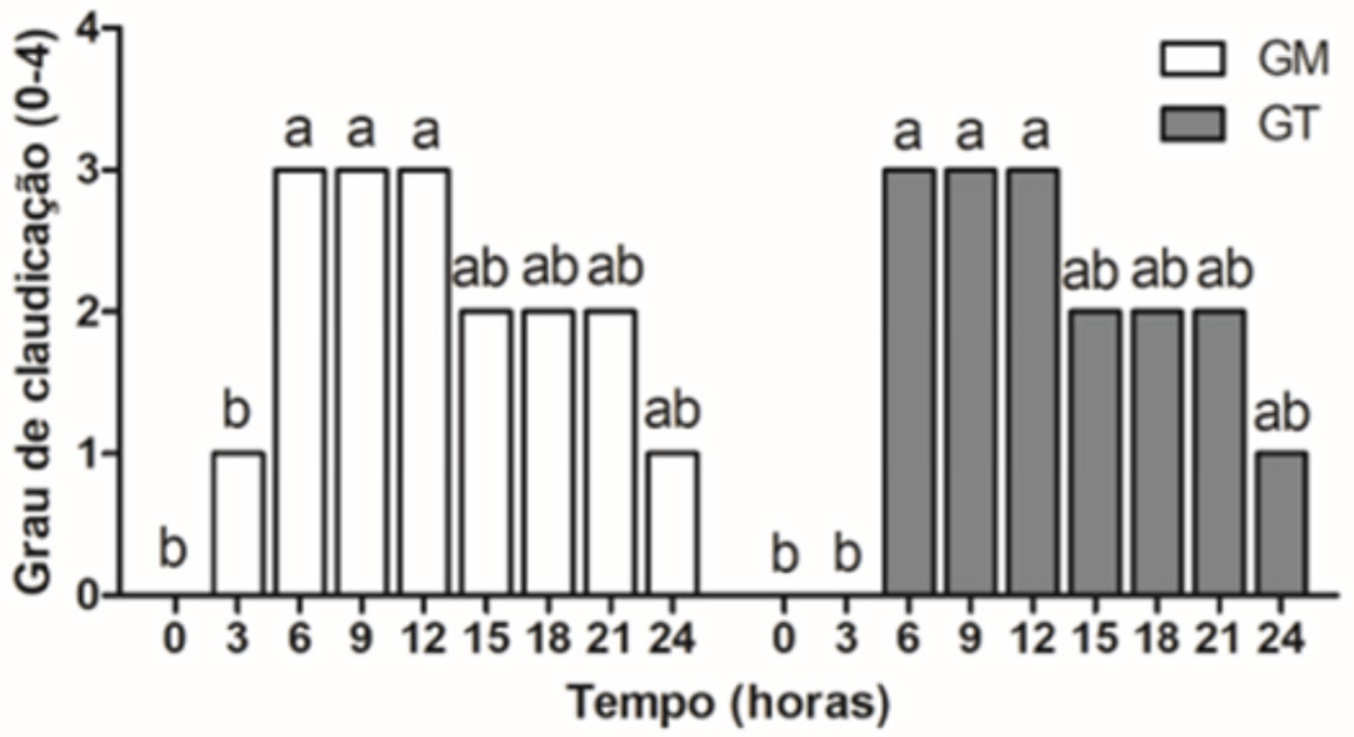

Figura 2. Mediana do grau de claudicação, segundo o grupo e tempo de avaliação. ${ }^{a b}$ Medianas seguidas de letras distintas na coluna diferem entre si pelo teste de Tukey $(\mathrm{P}<0,05)$ Não existe diferença significativa entre os grupos $(P>0,05)$

Tabela 2. Média $(\bar{x})$ e desvio padrão (S) do número de decúbitos e do tempo de decúbito, em minutos, segundo o grupo e tempo de avaliação

\begin{tabular}{|c|c|c|c|c|}
\hline \multirow{3}{*}{ Tempo (h) } & \multicolumn{2}{|c|}{ Número de decúbitos } & \multicolumn{2}{|c|}{ Tempo de decúbito (min) } \\
\hline & GM & GT & GM & GT \\
\hline & $\bar{x} \pm \mathrm{S}$ & $\bar{x} \pm \mathbf{S}$ & $\bar{x} \pm \mathbf{S}$ & $\bar{x} \pm \mathbf{S}$ \\
\hline 0 & $2 \pm 0,5^{\mathrm{ab}}$ & $2 \pm 0,5 \mathrm{ab}$ & $101 \pm 22^{2}$ & $97 \pm 17,4$ ab \\
\hline 3 & $0 \pm 0,0^{b}$ & $1 \pm 0,8 \mathrm{ab}$ & $0 \pm 0^{d}$ & $3 \pm 3,3 \mathrm{~d}$ \\
\hline 6 & $6 \pm 1,6^{a}$ & $3 \pm 2,1 a b$ & $56 \pm 31 \mathrm{abc}$ & $32 \pm 23,4$ cd \\
\hline 9 & $1 \pm 0,8 \mathrm{ab}$ & $3 \pm 1,7^{2}$ & $36 \pm 46$ bed & $70 \pm 31,5^{b}$ \\
\hline 12 & $1 \pm 0,4 \mathrm{ab}^{*}$ & $3 \pm 1,2$ ab & $19 \pm 19 \mathrm{~cd}^{*}$ & $83 \pm 22,1$ ab \\
\hline 15 & $0 \pm 0,5^{b}$ & $1 \pm 0,5^{b}$ & $17 \pm 40 \mathrm{~cd}$ & $4 \pm 5,9 d$ \\
\hline 18 & $0 \pm 0,4^{b}$ & $1 \pm 0,4 a b$ & $1 \pm 1 \mathrm{~cd}^{*}$ & $79 \pm 23,4^{a b}$ \\
\hline 21 & $1 \pm 0,5 \mathrm{ab}$ & $2 \pm 0,5 \mathrm{ab}$ & $86 \pm 45 \mathrm{abc}$ & $109 \pm 17,2^{2}$ \\
\hline 24 & $1 \pm 1,1$ ab & $1 \pm 0,4 a b$ & $15 \pm 12 \mathrm{~cd}^{*}$ & $65 \pm 13,4$ bc \\
\hline
\end{tabular}

${ }^{\text {bb }}$ Médias seguidas de letras distintas na coluna diferem entre si pelo teste de Tukey $(\mathrm{P}<0,05)$

- Existe diferença significativa entre os grupos $(\mathrm{P}<0,05)$ 
Considerando o tempo de decúbito (Tabela 2), houve diferença entre os grupos nos momentos de 6, $12,18,21$ e 24 horas, sendo os valores do GT significativamente maiores, exceto em 6 horas.

Avaliando-se os tempos nos grupos, observou-se que no GM ocorreu em zero hora o maior valor apresentado para o posicionamento em minutos, ou seja, no valor determinado como padrão, estando todos os demais momentos reduzidos em relação a esse. O menor valor encontrado para variável, representando a ausência de decúbito, ocorreu em três horas, seguido dos momentos de 12, 15, 18 e 24 horas.

No GT, o maior tempo de decúbito foi observado em 21 horas, seguido do tempo zero e os menores valores ocorreram em três e 15 horas.

Para variável número de passos (Tabela 3), observou-se diferença entre GM e GT em três, 15, 18, 21 e 24 horas.

Tabela 3. Média $(\bar{x})$ e desvio padrão (S) do número de passos e do número de toques ao solo, segundo o grupo e tempo de avaliação

\begin{tabular}{|c|c|c|c|c|}
\hline \multirow{3}{*}{ Tempo (h) } & \multicolumn{2}{|c|}{ Número de passos } & \multicolumn{2}{|c|}{ Tempo de toques ao solo } \\
\hline & GM & GT & GM & GT \\
\hline & $\bar{x} \pm \mathbf{S}$ & $\bar{x} \pm \mathrm{S}$ & $\bar{x} \pm \mathbf{S}$ & $\bar{x} \pm \mathbf{S}$ \\
\hline 0 & $1810 \pm 349^{a}$ & $1389 \pm 260^{a}$ & $383 \pm 78^{c}$ & $413 \pm 86$ bcd \\
\hline 3 & $1063 \pm 219 \mathrm{ab}^{*}$ & $651 \pm 205$ ab & $881 \pm 147 \mathrm{a}^{*}$ & $473 \pm 206 b c$ \\
\hline 6 & $1075 \pm 404 a b$ & $1042 \pm 342^{a b}$ & $887 \pm 109^{a}$ & $889 \pm 49$ a \\
\hline 9 & $585 \pm 186^{b}$ & $416 \pm 141^{b}$ & $544 \pm 148$ be & $351 \pm 86^{c d}$ \\
\hline 12 & $584 \pm 114^{b}$ & $570 \pm 323 \mathrm{ab}$ & $592 \pm 73 b c$ & $462 \pm 186 b c$ \\
\hline 15 & $1825 \pm 494^{a^{*}}$ & $716 \pm 98 \mathrm{ab}$ & $649 \pm 248 a b$ & $607 \pm 97^{b}$ \\
\hline 18 & $665 \pm 140^{b^{*}}$ & $388 \pm 60^{b}$ & $570 \pm 126^{b c^{*}}$ & $313 \pm 61$ cd \\
\hline 21 & $998 \pm 387 \mathrm{ab}^{*}$ & $327 \pm 85^{b}$ & $653 \pm 93 \mathrm{ab}^{*}$ & $215 \pm 34^{d}$ \\
\hline 24 & $686 \pm 169^{b *}$ & $378 \pm 82^{b}$ & $583 \pm 147 \mathrm{cc}^{*}$ & $343 \pm 114$ cd \\
\hline
\end{tabular}

${ }^{2 b}$ Médias seguidas de letras distintas na coluna diferem entre si pelo teste de Tukey $(\mathrm{P}<0,05)$

- Existe diferença significativa entre os grupos $(\mathrm{P}<0,05)$

No GM houve diferença significativa entre os tempos avaliados, sendo observados os maiores valores no tempo zero (medida padrão) e 15 horas. Os menores valores foram encontrados em 9, 12, 18 e 24 horas.

Comparando-se as medianas do número de passos, após a administração de morfina em seis horas, observou-se que, neste primeiro momento houve redução quando comparado ao valor padrão, assim como estabilidade quando comparada ao tempo de três horas, sendo estes valores inferiores ao tempo zero. Observou-se ainda, uma grande redução no momento seguinte, em nove horas.

Após a segunda aplicação de morfina, em 12 horas, também houve redução, encontrando-se nesse 
tempo, o menor valor registrado para a variável. Notou-se o inverso do primeiro momento de tratamento e ainda registrou-se, após a segunda aplicação, o maior valor de número de passos verificado às 15 horas.

Para o GT o valor padrão também demonstrou ser o maior registrado no tempo zero. As menores aferições foram observadas em 9, 18, 21 e 24 horas. As variáveis se mantiveram estabilizadas em valores intermediários nos momentos três, 6,12 e 15 horas.

Excetuando-se o momento de 15 horas do GM, todos os demais valores de ambos os grupos se apresentaram inferiores aos seus padrões em zero hora.

A análise da Tabela 3 evidencia diferença significativa entre os grupos nos momentos três, 18, 21 e 24 horas, considerando o número de toques no solo.

Comparando-se os dados do GM, observou-se os maiores valores do número de toques no solo em três e seis horas, em que o segundo momento se refere a uma hora após a administração do fármaco. O menor valor foi registrado em zero hora (padrão).

No GT, o valor padrão apresentou-se em uma zona intermediária, em que o maior número de toques também foi registrado em seis horas e o menor em 21 horas.

\section{Discussão}

A mensuração dos parâmetros fisiológicos das frequências cardíaca, respiratória e motilidade ruminal, não apresentou relevância para o estudo. Provavelmente, devido aos efeitos de estresse ambiental e manejo.

Baniadam et al. ${ }^{(5)}$, da mesma forma, não encontraram alterações significativas nas frequências cardíaca e respiratória, em avaliação dos efeitos da aplicação epidural de tramadol em bovinos, nas doses de $1,0 \mathrm{mg} / \mathrm{kg}, 2,0 \mathrm{mg} / \mathrm{kg}$ e $3,0 \mathrm{mg} / \mathrm{kg}$. Relataram ainda manutenção da motilidade ruminal normal. Porém, no presente estudo, observou-se redução dos movimentos ruminais em todos os momentos. A manipulação e contenção, mesmo submetendo os animais a um período de adaptação, parecem ter tido efeito marcante sobre estas variáveis.

Apesar dos animais de ambos os grupos claudicarem até o término do experimento, houve redução espontânea do escore de claudicação próximo ao final do período de avaliação. Ocorreu o retorno à deambulação normal após 72 horas em todos os animais, sem quaisquer sequelas locomotoras ${ }^{(11)}$.

O máximo de claudicação para este estudo foi o grau 3, que pôde ser observado entre seis e 12 horas, concomitante ao período do pico de estimulação dolorosa determinado pelo protocolo de indução de artrite e sinovite, desencadeado pela anfotericina B intra-articular ${ }^{(3)}$. Por meio desta manifestação, verificou-se a ausência de melhora da deambulação sob a ação da morfina ou tramadol no referido momento. Apesar do tempo de ação analgésica da morfina, para bovinos, de seis horas ${ }^{(12)}$ e meia-vida de quatro horas ${ }^{(12)}$, e intervalo de administração do tramadol determinado por alometria, em função da falta de dados para espécie.

A queda dos escores de claudicação ocorreu somente após as 12 horas em ambos os grupos, sugerindo uma melhora em função da diminuição do estímulo doloroso, que foi induzido em caráter transitório. 
A pedometria utilizada no presente estudo permitiu a observação da redução no número de passos, nos grupos GM e GT. Esta observação se fez comparando o momento inicial da avaliação e às 12 horas seguintes a indução do processo inflamatório articular, momento em que os animais apresentaram o máximo de estimulação dolorosa e manifestação de claudicação grau 3.

Em relação ao número de toques ao solo (passos falsos), mensurados pelos pedômetros, observou-se os maiores valores desta variável no momento correspondente ao de maior desenvolvimento de dor podal, concomitante ao início do pico de claudicação, em seis horas.

Analisando-se a variável tempo de decúbito, observou-se que o intervalo de tempo com maior demonstração de claudicação, ocorreu entre seis e 12 horas, havendo redução do tempo de decúbito no GM, apesar dos animais terem deitado mais vezes em seis horas. No GT também se observou esta alteração, ocorrendo diminuição do tempo em seis e nove horas, estabilizando estatisticamente em 12 horas. O mesmo comportamento foi notado em relação ao número de decúbitos no GT, uma vez que em nove horas houve aumento em relação ao momento inicial.

Estes achados da pedometria demonstram claramente uma inquietação dos animais, observada pelo aumento de toques ao solo, redução do número de passos, diminuição do tempo de decúbito e aumento do número de vezes nesta postura, nos momentos de evidência de claudicação grau 3 , podendo ser interpretado como demonstração de dor podal ${ }^{(11)}$.

Braz et al. (2) utilizando-se do tramadol nas doses de 2 e $4 \mathrm{mg} / \mathrm{kg}$, via intravenosa e nas doses de 100 e $200 \mathrm{mg}$, via retal, não observaram efeito analgésico, frente ao estímulo de dor a partir de descorna cáustica em bezerros. O mesmo achado foi visto em nosso estudo, com a administração do fármaco via intramuscular, na dose de $1,8 \mathrm{mg} / \mathrm{kg}$, alterando-se o método de estimulação dolorosa.

Anderson e Edmondson ${ }^{(6)}$ indicam a morfina na dose de $0,5 \mathrm{mg} / \mathrm{kg}$, via intravenosa, a cada 12 horas, como terapia analgésica em bovinos. Contudo, após a indução do complexo artrite e sinovite interfalângica distal, o mesmo fármaco na dose de $0,5 \mathrm{mg} / \mathrm{kg}$, mostrou-se incapaz de interferir na atenuação do grau de claudicação, frente à estimulação dolorosa experimental.

Foram empregados dois fármacos analgésicos, após estimulação dolorosa podal nos bovinos. Um deles, a morfina, de utilização ampla e consagrada na espécie. Apesar disso, nos resultados deste trabalho, baseando-se na qualidade de deambulação e atividade pedométrica, observou-se uma contraposição às indicações em literatura consultada ${ }^{(4,6)}$. Assim, evidencia-se que a terapia com morfina, na dose utilizada, foi incapaz atenuar o estímulo doloroso. Essa observação está respaldada pelo aumento da variável representada pelo número de toques ao solo, diminuição do número de passos, aumento do número de decúbitos e diminuição do tempo de decúbitos, coletados a partir da pedometria. Além da prevalência de manifestação clínica de claudicação grau 3, nos momentos de maior estimulação dolorosa do modelo experimental, apesar da terapia analgésica.

Apesar de o tramadol não possuir uma dose terapêutica definida para espécie bovina, o referido fármaco também apresentou resultados insatisfatórios na dose utilizada, frente ao estímulo doloroso a partir dos processos inflamatórios articulares, corroborando Braz et al. ${ }^{(2)}$. Todavia, é necessário um questionamento e avaliação do tramadol para essa espécie, verificando a farmacocinética, conforme descrito por Sousa et al. ${ }^{(13)}$ em caprinos, que apontaram a inexistência do principal metabólito (M1) no plasma, após administração de tramadol via oral na dose de $2 \mathrm{mg} / \mathrm{kg}$.

\section{Conclusões}


A morfina e o tramadol, nas doses de 0,5 e $1,8 \mathrm{mg} / \mathrm{kg}$, respectivamente, administrados por via intramuscular, foram incapazes de interferir na atenuação do grau de claudicação no momento de máxima estimulação dolorosa, frente ao modelo experimental de dor ortopédica.

\section{Referências}

1. Schulz KL, Anderson DE, Coetzee JF, White BJ, Miesner MD. Effect of flunixin meglumine on the amelioration of lameness in dairy steers with amphotericin B - induced transient synovitis - arthritis. Am J Vet Res. 2011; 72: 1431-1438.

2. Braz MR, Carreira M, Carolino N, Rodrigues T, Stilwell G. Effect of rectal or intravenous tramadol on the incidence of pain-related behaviour after disbudding calves with caustic paste. Appl Anim Behav Sci. 2012; 136(1): 20-25.

3. Kotschwar JL, Coetzee JF, Anderson DE, Gehring R, Kukanich B, Apley MD. Analgesic efficacy of sodium salicylate in an amphotericin B - induced bovine synovitis - arthritis model. J Dairy Sci. 2009; 92: 3731-3743.

4. Anderson DE, Muir WW. Pain management in cattle. Vet Clin North Am Food Anim Pract. 2005; 21: 623635.

5. Baniadam A, Afshar FS, Ahmadian F. Analgesic effects of tramadol hydrochlride administered via caudal epidural injection in healthy adult cattle. Am J Vet Res. 2010; 71: 720-725.

6. Anderson DE, Edmondson MA. Prevention and management of surgical pain in cattle. Vet Clin North Am Food Anim Pract. 2013; 29: 157-184.

7. Desrochers A, Anderson DE, St-Jean G. Lameness examination in cattle. Vet Clin North Am Food Anim Pract. 2001; 17: 39-52.

8. Mazrier H, Tal S, Aizinbud E, Bargai U. A field investigation of the use of the pedometer for the early detection of lameness in cattle. Can Vet J. 2006; 47: 883-886.

9. Pachaly JR. Terapêutica por extrapolação alométrica. In: Cubas ZS, Silva JCR, Catão-Dias JL. Tratado de animais selvagens. São Paulo: Roca; 2006. 1215-1223.

10. Feitosa FLF. Semiologia do sistema digestório de ruminantes. In: . Semiologia - A arte do diagnóstico. São Paulo: Roca; 2008. 90-117.

11. Shearer JK, Amstel SRV, Coetzee JF. Assessment and management of pain associated with lameness in cattle. Vet Clin North Am Food Anim Pract. 2013; 29: 135-156.

12. Górniak SL. Hipnoanalgésicos. In: Spinosa HS, Górniak SL, Bernardi MM. Farmacologia aplicada à medicina veterinária. Rio de Janeiro: Guanabara Koogan; 2017. 184-194.

13. Sousa AB, Santos ACD, Schramm SG, Porta V, Górniak SL, Florio JC, Spinosa HDS. Pharmacokinetics of tramadol and o-desmethyltramadol in goats after intravenous and oral administration. J Vet Pharmacol Therap. 2008; 31: 45-51. 